\title{
Gene Expression Pathways across Multiple Tissues in Antineutrophil Cytoplasmic Antibody-associated Vasculitis Reveal Core Pathways of Disease Pathology
}

\author{
Marcia A. Friedman (1), Dongseok Choi $(\mathbb{D}$, Stephen R. Planck (iD, James T. Rosenbaum (1), \\ and Cailin H. Sibley
}

ABSTRACT. Objective. To identify commonalities in gene expression data across all antineutrophil cytoplasmic antibody (ANCA)-associated vasculitis (AAV) tissues thus far characterized.

Methods. Gene expression data were collected from the 3 AAV tissues thus far characterized (orbit, peripheral leukocytes, and sinus brushings). These data were analyzed to identify commonly expressed genes and disease pathways. The pathways data were adjusted for multiple comparisons using a combined local false discovery rate, which estimates the probability of a false discovery of a given pathway in all 3 tissues analyzed.

Results. Only 4 genes were upregulated in all 3 tissues - IL1RN,TLR2, SLC11A1, and MMP9. After multiple comparison adjustments, the network pathway analysis revealed 28 pathways associated with all 3 tissues. The most strongly associated pathway for all 3 tissues was the neutrophil degranulation pathway [multidimensional local false discovery (md-locfdr) $=1.05 \times 10^{-12}$ ], followed by the osteoclast differentiation (md-locfdr $\left.=3.8 \times 10^{-05}\right)$, cell surface interactions at the vascular wall $\left(\right.$ md-locfdr $\left.=4.2 \times 10^{-04}\right)$, signaling by interleukins $\left(\right.$ md-locfdr $\left.=6.1 \times 10^{-04}\right)$, and phagosome $(\mathrm{md}-$ locfdr $=0.003$ ) pathways. There were no downregulated genes or pathways common to all 3 tissues.

Conclusion. This analysis identified individual genes and pathways of disease common to all AAV tissues thus far characterized. The use of a network pathway analysis allowed us to identify pathologic mechanisms that were not readily apparent in the commonly expressed genes alone. Many of these pathways are consistent with current theories about infectious drivers and the crossroads of innate and adaptive immune mechanisms. In addition, this analysis highlights novel pathways, such as vessel wall interactions and platelet activation, which require further investigation. (First Release January 15 2019; J Rheumatol 2019;46:609-15; doi:10.3899/jrheum.180455)

\section{Key Indexing Terms: \\ ANTINEUTROPHIL CYTOPLASMIC ANTIBODY-ASSOCIATED VASCULITIS \\ VASCULITIS GRANULOMATOSIS WITH POLYANGIITIS METAANALYSIS MICROSCOPIC POLYANGIITIS GENE EXPRESSION}

From the Department of Medicine, and the Casey Eye Institute, and the OHSU-PSU (Portland State University) School of Public Health, Oregon Health \& Science University (OHSU), Portland, Oregon, USA; Graduate School of Dentistry, Kyung Hee University, Seoul, Korea; Devers Eye Institute, Legacy Health Systems, Portland, Oregon, USA.

This research was supported by the US National Institutes of Health (NIH) National Institute of Arthritis and Musculoskeletal and Skin Diseases: 5K23AR068440; the NIH National Heart, Lung, and Blood Institute: 3T32HL094294-08S1; the NIH National Eye Institute: EY010572, EY020249, and EY265722; Research to Prevent Blindness; the Stan and Madelle Rosenfeld Family Trust; the William and Mary Bauman Foundation.

M.A. Friedman, MD, Instructor of Medicine, Department of Medicine, OHSU; D. Choi, PhD, Professor, Department of Medicine, and the Casey Eye Institute, and the OHSU-PSU School of Public Health, and the Graduate School of Dentistry, Kyung Hee University; S.R. Planck, PhD, Professor of Ophthalmology, Department of Medicine, and Casey Eye Institute, OHSU; J.T. Rosenbaum, MD, Professor of Ophthalmology, Department of Medicine, and Casey Eye Institute, OHSU, and Devers Eye Institute, Legacy Health Systems; C.H. Sibley, MD, Assistant Professor of Medicine, Department of Medicine, OHSU.

Address correspondence to Dr. M.A. Friedman, Division of Arthritis \& Rheumatology, Oregon Health \& Science University, 3181 SW Sam Jackson Park Road, Portland, Oregon 97008, USA.E-mail:friedmam@ohsu.edu Accepted for publication October 11, 2018.
Granulomatosis with polyangiitis (GPA) and microscopic polyangiitis (MPA) are life-threatening multisystem vasculitides affecting small to medium-sized blood vessels. Both diseases are associated with the antineutrophil cytoplasmic antibody (ANCA) and are together referred to as ANCA-associated vasculitis (AAV). Several studies have characterized the gene expression signature of AAV in affected tissues. To date, the gene expression signatures of orbital tissue, peripheral leukocytes, and nasal sinus brushings have been reported ${ }^{1,2,3}$. The purpose of our study is to compare gene expression data across these 3 tissues to discover common AAV disease pathways and to validate reported data.

Commonalities in gene expression data can be determined by focusing on individual genes. However, the comparison of pathways can lend additional insight beyond the gene level alone. The comparative pathway analysis approach has been used in metaanalyses of cancers, systemic lupus erythematosus (SLE), rheumatoid arthritis, ankylosing spondylitis, neuropsychiatric disorders, and other diseases ${ }^{4,5,6,7,8,9,10}$.

Personal non-commercial use only. The Journal of Rheumatology Copyright @ 2019 . All rights reserved. 
Individual genes have many functions and can affect a large number of different processes. Disease pathways involve many genes that may be used at different points in time in different tissues. In the study of SLE, for example, pathway analyses identified the importance of the interferon pathway based on interferon-inducible genes, even though the interferons themselves were not upregulated ${ }^{9}$. Through the use of a network analysis system based on knowledge of protein-protein interactions, network pathway analyses allow association of the biologic functions and pathologic mechanisms with a set of expressed genes.

In this analysis, we tested the hypothesis that there are commonly preserved pathways of disease associated with all tissues thus far characterized in AAV. The pathway analysis yielded more robust pathogenic information than the evaluation of commonly expressed individual genes alone. The identified pathways reflect known and plausible mechanisms of AAV disease.

\section{MATERIALS AND METHODS}

Study inclusion criteria. We used published gene expression data from studies of patients with AAV compared to controls. Studies were identified through a PubMed search for studies containing at least $1 \mathrm{AAV}$ term (ANCA, GPA, MPA, or Wegener's) and at least 1 gene expression term (gene expression, RNA, or microarray). Ethics and institutional review board approval was not required because our study is a metaanalysis of previously published and de-identified data.

The set of differentially upregulated and then downregulated genes from each study was analyzed by the Cytoscape software application ${ }^{11,12}$ with the Reactome Functional Interactional (FI) plugin ${ }^{12}$. This is a pathway-based network analysis system that uses expert-gathered pathways based on available knowledge of protein-protein interactions. The 2016 Reactome FI application incudes 1127 pathways collected from Reactome as well as other pathway databases including the National Cancer Institute Pathway Interactions Database, Kyoto Encyclopedia of Genes and Genomes, Panther, CellMap, and Biocarta ${ }^{13,14}$.

Pathways associated with upregulated gene lists from each tissue were collected along with (the conventional Benjamini-Hochberg global) false discovery rate (FDR) and $p$ values ${ }^{15}$. However, because there is no existing method to combine 3 lists of FDR directly, we chose to estimate the local FDR (locfdr) for pathways of each tissue and multidimensional locfdr (md-locfdr) of common pathways of 3 tissues ${ }^{16}$. The locfdr and md-locfdr of pathway lists were estimated using a semiparametric mixture model ${ }^{16}$. A locfdr is an estimate of the probability of an individual pathway being a false discovery in that tissue, while the md-locfdr can be thought of as the probability of an individual pathway being a false discovery in all 3 tissues. The locfdr and the conventional FDR are closely related. A locfdr can be defined as $\operatorname{locfdr}(\mathrm{z})=\operatorname{Probability}\{$ null1 $\mathrm{Z}=\mathrm{z}\}$, where $\mathrm{Z}$ is a test statistic, and the conventional $\operatorname{FDR}$ as $\operatorname{FDR}(\mathrm{z})=\mathrm{E}\{\operatorname{fdr}(\mathrm{Z}) \mid \mathrm{Z} \leq \mathrm{z}\}$. That is, $\operatorname{FDR}(\mathrm{z})$ is a (weighted) average of locfdr, $\operatorname{locfdr}(\mathrm{Z})$ for $\mathrm{Z} \leq \mathrm{z}$. The technical details of locfdr can be found in Supplement A (available with the online version of this article) and in Jeong, $e t a l^{16}$. While there is no widely accepted cutoff value for locfdr, Efron suggested that a locfdr of 0.2 could be used, which corresponds to the conventional FDR of 0.05 to 0.15 under some mild conditions ${ }^{17}$. In our study, an locfdr of $<0.2$ was used as a cutoff. Twenty-eight pathways with an md-locfdr of $<0.2$ were identified.

Downregulated genes were those with a fold change (FC) value of $\leq-1.5$ in ANCA samples compared to healthy controls. A conventional FDR cutoff of $<0.25$ was used to identify pathways associated with the downregulated gene lists. Because very few pathways were identified and none were associated with all 3 tissues, locfdr was not used for this analysis.

\section{RESULTS}

Three studies were identified that characterized the gene expression of AAV tissues compared to healthy controls. This included studies of peripheral leukocytes ${ }^{3}$, sinus brushings ${ }^{2}$, and orbital inflammatory disease ${ }^{1}$. All 3 studies used RNA microarray to characterize these tissues. The characteristics of each study are shown in Table 1. Differences in treatment with glucocorticoid and other immunosuppressant medications are not included in the table; however, the nasal sinus brushing and orbital inflammatory data were adjusted for medication use. Medication use in the peripheral leukocyte study is not precisely known. The nasal sinus brushing and orbital inflammatory disease papers included only patients with GPA. The peripheral leukocyte study included patients who had AAV with pauciimmune glomerulonephritis; however, the AAV subtype was not reported ${ }^{3}$.

Upregulated genes in common. We identified only 4 genes that were upregulated across all 3 studies: interleukin 1 receptor antagonist $(I L I R N)$, matrix metalloproteinase 9 (MMP9), solute carrier family 11 member 1 (SLC11A1), and Toll-like receptor 2 (TLR2). These 4 genes were identified in all 3 tissues with an FC compared to control tissues of $>1.5$ and $\mathrm{p}$ values of $<0.05$.

$I L I R N$ is upregulated in peripheral leukocytes $(\mathrm{FC}=1.7$, $\left.\mathrm{p}=1.9 \times 10^{-4}\right)$, sinus tissue $\left(\mathrm{FC}=2.5, \mathrm{p}=1.9 \times 10^{-4}\right)$, and orbital tissue $\left(\mathrm{FC}=3.69\right.$ and $4.06, \mathrm{p}=4.68 \times 10^{-3}$ and 8.97 $\left.\times 10^{-3}\right)$. The orbital study shows 2 values each for FC and $\mathrm{p}$ values because in our study, 2 sets of samples were processed at different timepoints. SLC11A1 encodes an iron transport membrane protein and is upregulated in peripheral leukocytes $\left(\mathrm{FC}=2.3, \mathrm{p}=8.4 \times 10^{-7}\right)$, sinus tissue $(\mathrm{FC}=2.5, \mathrm{p}=1.9 \times$ $\left.10^{-4}\right)$, and orbital tissue ( $\mathrm{FC}=3.5$ and $3.3, \mathrm{p}=3.6 \times 10^{-4}$ and $1.9 \times 10^{-2}$ ). TLR2 encodes a cell surface protein of the TLR family; it is upregulated in peripheral leukocytes $(\mathrm{FC}=2.2$, $\left.\mathrm{p}=2.1 \times 10^{-6}\right)$, sinus tissue $\left(\mathrm{FC}=1.7, \mathrm{p}=2.3 \times 10^{-3}\right)$, and orbital tissue $\left(\mathrm{FC}=3.1 \text { and } 2.5, \mathrm{p}=3.3 \times 10^{-2} \text { and } 2.6 \times 10^{-2}\right)^{18}$. $M M P 9$ is upregulated in peripheral leukocytes $(\mathrm{FC}=4.0$, $\left.\mathrm{p}=1.5 \times 10^{-6}\right)$, nasal tissue $\left(\mathrm{FC}=2.2, \mathrm{p}=2.0 \times 10^{-4}\right)$, and the orbit $\left(\mathrm{FC}=10.4\right.$ and $4.75, \mathrm{p}=2.2 \times 10^{-5}$ and $\left.1.5 \times 10^{-2}\right)$. A further 45 genes were identified that were present in at least 2 of the 3 tissues; these are listed in Table 2 .

Associated upregulated pathways in common. Out of 1127 identifiable pathways, 28 had an md-locfdr of $<0.2$ across all 3 tissues ${ }^{16,17}$. These 28 disease pathways are represented in Table 3. The individual genes associated with each pathway in each tissue are shown in the Supplementary Table 1 (available with the online version of this article).

The 28 pathways discovered in this analysis can be grouped into 8 functional categories including innate immunity, adaptive immunity, vascular wall interactions, cellular signaling, complement activation, tissue damage/repair, infectious disease pathways, and platelet activation (Table 3 ). The most strongly associated pathway in all 3 tissues is the neutrophil degranulation pathway $\left(\right.$ locfdr $\left.=1.05 \times 10^{-12}\right)$.

Personal non-commercial use only. The Journal of Rheumatology Copyright $\odot$ (2019. All rights reserved 


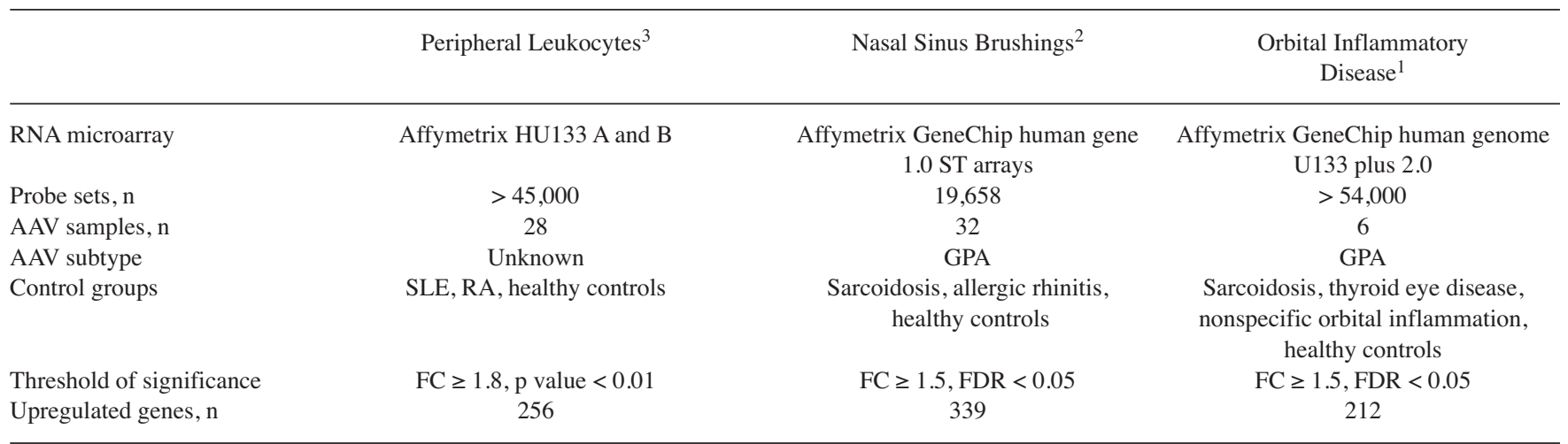

FC: fold change; AAV: antineutrophil cytoplasmic antibody-associated vasculitis; FDR: false discovery rate; GPA: granulomatosis with polyangiitis; SLE: systemic lupus erythematosus; RA: rheumatoid arthritis.

Table 2. Individual genes upregulated in 2 or more AAV studies.

\begin{tabular}{|c|c|c|c|c|c|}
\hline \multirow{2}{*}{$\begin{array}{l}\text { Studies } \\
\text { Genes in common }\end{array}$} & \multicolumn{2}{|c|}{ Orbit and Nasal Sinus Brushings } & \multirow{2}{*}{$\begin{array}{c}\begin{array}{c}\text { Orbit and } \\
\text { Leukocytes }\end{array} \\
\text { ILIRN }\end{array}$} & \multicolumn{2}{|c|}{$\begin{array}{l}\text { Nasal Sinus Brushings } \\
\text { and Leukocytes }\end{array}$} \\
\hline & $I L I R N$ & FN1 & & $I L I R N$ & $M M P 25$ \\
\hline & $M M P 9$ & ITGAX & $M M P 9$ & $M M P 9$ & NFE2 \\
\hline & SLC11A1 & $I T G B 2$ & SLC11A1 & SLC11A1 & NFIL3 \\
\hline & $T L R 2$ & LILRB3 & $T L R 2$ & $T L R 2$ & OSM \\
\hline & $A D A M 8$ & $N L R P 3$ & SLC8A1 & $A B C A 1$ & PFKFB3 \\
\hline & $A L O X 5$ & SERPINA1 & & ACSL1 & PROK2 \\
\hline & CHI3L1 & $S P P 1$ & & $A D M$ & $Q P C T$ \\
\hline & CLEC4D & $T L R 8$ & & АРОВЕС $3 A$ & RASGRP4 \\
\hline & CLEC7A & TREML2 & & $B C L 2 A 1$ & S100A12 \\
\hline & CXCR4 & & & $C D A$ & SAMSN1 \\
\hline & & & & DEFA3 & $S L C 2 A 3$ \\
\hline & & & & $D Y S F$ & SOCS3 \\
\hline & & & & EMR2 & SSH2 \\
\hline & & & & $\begin{array}{l}I L 18 R A P \\
I L 1 R 2\end{array}$ & TNFAIP6 \\
\hline
\end{tabular}

AAV: antineutrophil cytoplasmic antibody-associated vasculitis.

After this, the most strongly associated pathways are osteoclast differentiation (locfdr $=3.8 \times 10^{-05}$ ), cell surface interactions at the vascular wall (locfdr $=4.2 \times 10^{-04}$ ), signaling by interleukins $\left(\operatorname{locfdr}=6.1 \times 10^{-04}\right)$, and the phagosome (locfdr $=0.003)$.

Downregulated genes and pathways in common. There were no downregulated genes or pathways found in all tissues. Only 1 gene, the GOS 2 gene, was downregulated in 2 of the 3 tissues; peripheral leukocytes $(\mathrm{FC}=-1.9, \mathrm{p}=0.26)$, and the orbit $\left(\mathrm{FC}=-13.7\right.$ and $-24.9, \mathrm{p}=4.7 \times 10^{-3}$ and $1.4 \times$ $\left.10^{-4}\right)$. Downregulated pathways common to 2 out of 3 tissues are shown in Supplementary Table 2 (available with the online version of this article).

\section{DISCUSSION}

This is the first analysis of gene expression pathways in common across multiple tissues in AAV, to our knowledge. The use of a network analysis system has allowed us to identify biologic functions and pathologic mechanisms that were not readily apparent by analysis of upregulated genes alone. Many of the common genes and pathways identified in our analysis support current knowledge and theories about AAV pathophysiology, while other pathways have not previously been implicated in AAV, and thus they may offer novel insights into disease pathogenesis. The pathophysiology of AAV based on gene expression pathways is represented in Figure 19,20,21,22,23,24,25,26.

The results of our analysis support the central roles of an infectious trigger, molecular mimicry, and the dysregulation of both innate and adaptive immune mechanisms in AAV (Figure 1). Infections are thought to drive AAV through molecular mimicry. This is supported by the antimicrobial peptide pathway $\left(\right.$ locfdr $\left.=8.2 \times 10^{-03}\right)$, infectious disease pathways, and the clinical finding that nasal carriage of Staphylococcus aureus, which shares peptide homology with human proteinase 3 (PR3) antigen, increases the risk of

Personal non-commercial use only. The Journal of Rheumatology Copyright $\subset$ 2019. All rights reserved. 
Table 3. Pathways commonly associated with all ANCA-associated vasculitis gene expression studies.

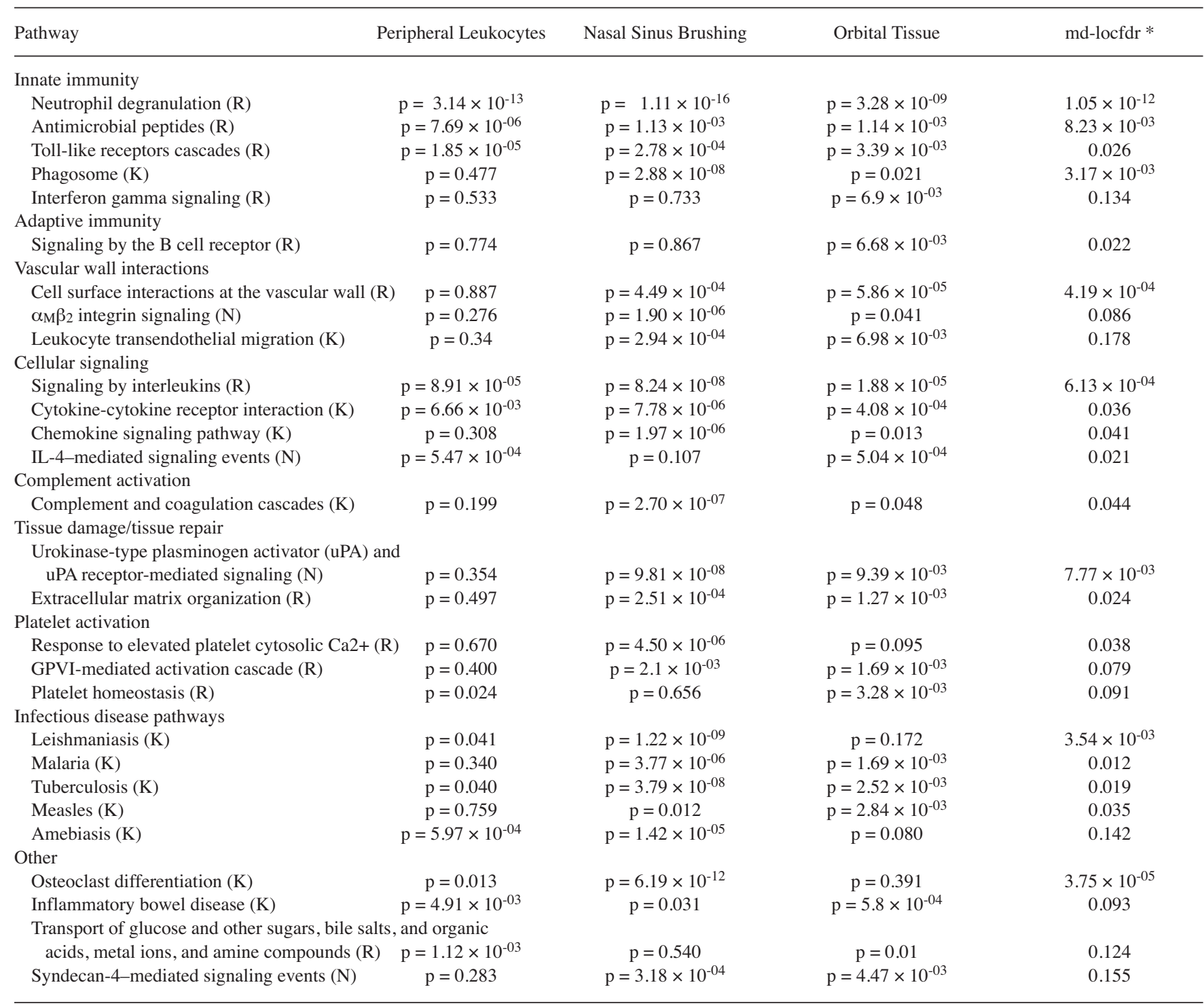

* md-locfdr: multidimensional local false discovery rate (probability of individual pathway being a false discovery in all 3 tissues). ANCA: antineutrophil cytoplasmic antibody; C: CellMap; R: Reactome; K: Kyoto Encyclopedia of Genes and Genomes; N: National Cancer Institute Pathway Interaction Database; IL: interleukin; GPVI: glycoprotein VI.

relapse in $\mathrm{GPA}^{20,27}$. Three of the four genes expressed in common, ILIRN, SLCIIA1, and TLR2, are also important innate immune genes. TLR2 is particularly interesting because it is the predominant TLR recognizing $S$. aureus ligands (Figure 1).

In addition to innate and infectious disease mechanisms, $\mathrm{AAV}$ is also an adaptive immune disease associated with pathogenic antibodies. Myeloperoxidase (MPO) antibodies have been found to be pathogenic in murine transfer models and in 1 transplacental human transfer case report ${ }^{28,29}$. However, perhaps the strongest evidence for ANCA pathogenicity is the success of rituximab treatment, which is increasingly becoming the standard of care in severe pathology ${ }^{30}$.
ANCA have diverse functions in this disease; however, their main function is activating primed neutrophils (Figure $1)^{23,31,32}$. All 3 tissues' gene lists were most strongly associated with the neutrophil degranulation pathway (locfdr $=1.05 \times 10^{-12}$ ). ANCA-activated neutrophils may degranulate or extrude their intracellular contents in the form of a neutrophil extracellular trap (NET) ${ }^{25,33}$. AAV neutrophils also activate the alternative complement cascade consistent with the complement and coagulation cascade pathway $($ locfdr $=0.044)$ in our analysis $(\text { Figure } 1)^{26}$. A C5a inhibitor is currently in clinical trials as a steroid-sparing agent in $\mathrm{AAV}^{26}$.

Platelet activation and vascular wall interaction pathways are not well understood regarding their role in AAV and 


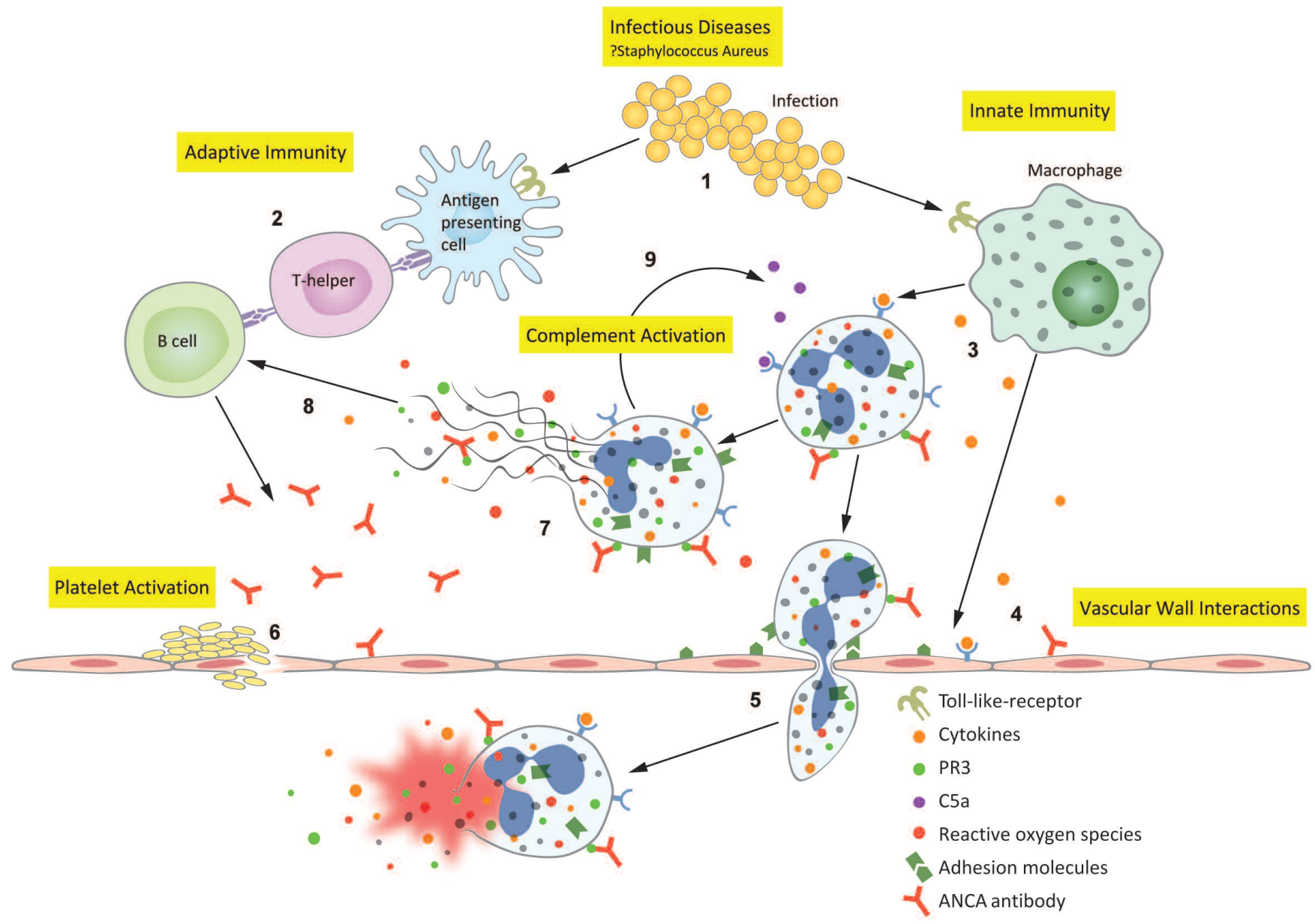

Figure 1. Antineutrophil cytoplasmic antibody (ANCA)-associated vasculitis pathophysiology revealed through gene expression pathways: 1. Infectious diseases, such as Staphylococcus aureus, activate Toll-like receptors on antigen presenting cells and macrophages ${ }^{19}$. 2 . Antimicrobial peptide homology with human antigens leads to the formation of pathogenic ANCA ${ }^{20}$. 3. Macrophages secrete inflammatory cytokines that prime neutrophils to express PR3 and MPO on their cell surface, allowing ANCA to bind and activate the neutrophil. 4. Cytokines and ANCA activate endothelial cells, which express leukocyte adhesion molecules ${ }^{21,22,23,24}$. 5. ANCA-activated neutrophils migrate through the vascular wall where they degranulate, releasing reactive oxygen species and damaging the vessel wall, thus causing the characteristic necrotizing vasculitis. 6. Platelets are activated at the sites of damaged endothelium; however, their precise contribution to AAV pathophysiology is not well understood. 7. Activated neutrophils release neutrophil extracellular traps (NET); NET contain all of the intracellular contents of a neutrophil including MPO and PR $3^{25}$. 8. Exposure of MPO and PR3 to the extracellular environment during NETosis drives further antibody formation. 9. Activated neutrophils also activate the alternative complement cascade, resulting in cleavage of C5 into C5a, and C5b-C5a goes on to prime and attract more neutrophils, leading to an amplification loop during active disease ${ }^{26}$. PR3: proteinase 3; MPO: myeloperoxidase; AAV: ANCA-associated vasculitis.

require further study. Patients with AAV are at increased risk for venous thrombosis, ischemic heart disease, and cardiovascular $(\mathrm{CV})$ events $^{34,35}$. Platelets are acute-phase reactants, but can also secrete cytokines and mediate inflammatory responses ${ }^{36,37}$. Despite the increased CV risk associated with $\mathrm{AAV}$, to date there are no specific recommendations for $\mathrm{CV}$ risk reduction in these patients.

The interaction of the peripheral immune mechanisms with the vascular wall represents an important gap in our understanding of this disease. Endothelial cells lining the vessel wall are heterogeneous and immunologically active cells, yet our understanding of how these cells function in AAV remains limited ${ }^{38}$. Enhancing our understanding of the vascular wall in AAV may allow us to understand patterns of organ involvement and to identify novel therapeutic targets.

Our analysis has limitations. Network-based pathway analyses are based on current knowledge of protein interactions, and some pathways may be associated owing to diverse functions of genes rather than specific AAV physiology. For example, while infections are likely important, pathogens such as malaria, amebiasis, and measles (Table 3) are unlikely to be the inciting pathogens. This analysis is also limited by variability in methodologies. Each study has a different number of samples and used a microarray with different numbers of probes, which may prevent the detection of more subtle pathways of interest. The control groups

Personal non-commercial use only. The Journal of Rheumatology Copyright @ 2019 . All rights reserved. 
studied for these tissues were also different, which may result in variability in which genes are considered differentially regulated when compared to controls. Finally, comparing peripheral leukocytes to end-organ tissue is problematic, because end-organ tissue includes a large number of other cell types that are not represented in the leukocyte study. Vascular wall interaction and tissue damage/repair pathways are more strongly associated with the orbital and nasal studies, which is most likely due to the inclusion of end-organ tissue in these studies.

Despite these limitations, our pathway analysis has successfully identified pathways of disease common to all AAV tissues thus far characterized. Many of these pathways are consistent with current theories about infectious drivers and the crossroads of innate and adaptive immune mechanisms. In addition, our analysis highlights novel pathways that require further investigation, such as vessel wall interactions and platelet activation. Further study of these pathways may lead to novel biomarkers and therapeutic strategies that improve the care of patients with this disease.

\section{ONLINE SUPPLEMENT}

Supplementary material accompanies the online version of this article.

\section{REFERENCES}

1. Rosenbaum JT, Choi D, Wilson DJ, Grossniklaus HE, Harrington $\mathrm{CA}$, Sibley $\mathrm{CH}$, et al. Orbital pseudotumor can be a localized form of granulomatosis with polyangiitis as revealed by gene expression profiling. Exp Mol Pathol 2015;99:271-8

2. Grayson PC, Steiling K, Platt M, Berman JS, Zhang X, Xiao J, et al. Defining the nasal transcriptome in granulomatosis with polyangiitis (Wegener's). Arthritis Rheumatol 2015;67:2233-9.

3. Alcorta DA, Barnes DA, Dooley MA, Sullivan P, Jonas B, Liu Y, et al. Leukocyte gene expression signatures in antineutrophil cytoplasmic autoantibody and lupus glomerulonephritis. Kidney Int 2007;72:853-64.

4. Xu W, Huang H, Yu L, Cao L. Meta-analysis of gene expression profiles indicates genes in spliceosome pathway are up-regulated in hepatocellular carcinoma (HCC). Med Oncol 2015;32:96.

5. Fang F, Pan J, Xu L, Li G, Wang J. Identification of potential transcriptomic markers in developing ankylosing spondylitis: A meta-analysis of gene expression profiles. Biomed Res Int 2015;2015:826316.

6. Lee YH, Bae SC, Song GG. Meta-analysis of gene expression profiles to predict response to biologic agents in rheumatoid arthritis. Clin Rheumatol 2014;33:775-82.

7. Yang Z, Chen Y, Fu Y, Yang Y, Zhang Y, Chen Y, et al. Meta-analysis of differentially expressed genes in osteosarcoma based on gene expression data. BMC Med Genet 2014;15:80.

8. Vosa U, Vooder T, Kolde R, Vilo J, Metspalu A, Annilo T. Meta-analysis of microRNA expression in lung cancer. Int J Cancer 2013;132:2884-93.

9. Arasappan D, Tong W, Mummaneni P, Fang H, Amur S. Meta-analysis of microarray data using a pathway-based approach identifies a 37-gene expression signature for systemic lupus erythematosus in human peripheral blood mononuclear cells. BMC Med 2011;9:65.

10. Gandal MJ, Haney JR, Parikshak NN, Leppa V, Ramaswami G, Hartl C, et al. Shared molecular neuropathology across major psychiatric disorders parallels polygenic overlap. Science 2018;359:693-7.
11. Shannon P, Markiel A, Ozier O, Baliga NS, Wang JT, Ramage D, et al. Cytoscape: A software environment for integrated models of biomolecular interaction networks. Genome Res 2003;13:2498-504.

12. Wu G, Feng X, Stein L. A human functional protein interaction network and its application to cancer data analysis. Genome Biol 2010;11:R53.

13. Fabregat A, Jupe S, Matthews L, Sidiropoulos K, Gillespie M, Garapati P, et al. The reactome pathway knowledgebase. Nucleic Acids Res 2018;46:D649-55.

14. ReactomeFIVIz. [Internet. Accessed December 11, 2018.] Available from: reactome.org/tools/reactome-fiviz

15. Benjamini Y, Hochberg Y. Controlling the false discovery rate: a practical and powerful approach to multiple testing. J R Stat Soc Series B Stat Methodol 1995;57:289-300.

16. Jeong SO, Choi D, Jang W. A semiparametric mixture method for local false discovery rate estimation. 2016. [Internet. Accessed December 11, 2018.] Available from: arxiv.org/abs/1604.04264

17. Efron B. Microarrays, empirical Bayes and the two-groups model. Statist Sci 2008;23:1-22.

18. Liu Y, Yin H, Zhao M, Lu Q. TLR2 and TLR4 in autoimmune diseases: A comprehensive review. Clin Rev Allergy Immunol 2014;47:136-47.

19. Uematsu S, Akira S. Toll-Like receptors (TLRs) and their ligands. Handb Exp Pharmacol 2008;183:1-20.

20. Tadema H, Heeringa P, Kallenberg CG. Bacterial infections in Wegener's granulomatosis: Mechanisms potentially involved in autoimmune pathogenesis. Curr Opin Rheumatol 2011;23:366-71.

21. Al-Hussain T, Hussein MH, Conca W, Al Mana H, Akhtar M. Pathophysiology of ANCA-associated vasculitis. Adv Anat Pathol 2017;24:226-34.

22. Taekema-Roelvink ME, Kooten C, Kooij SV, Heemskerk E, Daha MR. Proteinase 3 enhances endothelial monocyte chemoattractant protein-1 production and induces increased adhesion of neutrophils to endothelial cells by upregulating intercellular cell adhesion molecule-1. J Am Soc Nephrol 2001;12:932-40.

23. Mayet WJ, Schwarting A, Orth T, Duchmann R, Meyer zum Buschenfelde KH. Antibodies to proteinase 3 mediate expression of vascular cell adhesion molecule-1 (VCAM-1). Clin Exp Immunol 1996;103:259-67.

24. Arrizabalaga P, Sole M, Abellana R, Ascaso C. Renal expression of adhesion molecules in anca-associated disease. J Clin Immunol 2008;28:411-9

25. Soderberg D, Segelmark M. Neutrophil extracellular traps in ANCA-associated vasculitis. Front Immunol 2016;7:256.

26. Kallenberg CG, Heeringa P. Complement system activation in anca vasculitis: A translational success story? Mol Immunol 2015;68:53-6.

27. Salmela A, Rasmussen N, Tervaert JWC, Jayne DRW, Ekstrand A, European Vasculitis Study Group. Chronic nasal Staphylococcus aureus carriage identifies a subset of newly diagnosed granulomatosis with polyangiitis patients with high relapse rate. Rheumatology 2017;56:965-72.

28. Jennette JC, Xiao H, Falk R, Gasim AM. Experimental models of vasculitis and glomerulonephritis induced by antineutrophil cytoplasmic autoantibodies. Contrib Nephrol 2011;169:211-20.

29. Bansal PJ, Tobin MC. Neonatal microscopic polyangiitis secondary to transfer of maternal myeloperoxidase-antineutrophil cytoplasmic antibody resulting in neonatal pulmonary hemorrhage and renal involvement. Ann Allergy Asthma Immunol 2004;93:398-401.

30. Stone JH, Merkel PA, Spiera R, Seo P, Langford CA, Hoffman GS, et al. Rituximab versus cyclophosphamide for ANCA-associated vasculitis. N Engl J Med 2010;363:221-32.

31. Kettritz R. How anti-neutrophil cytoplasmic autoantibodies activate neutrophils. Clin Exp Immunol 2012;169:220-8.

32. Xiao H, Hu P, Falk RJ, Jennette JC. Overview of the pathogenesis of anca-associated vasculitis. Kidney Dis (Basel) 2016;1:205-15. 
33. Grayson PC, Carmona-Rivera C, Xu L, Lim N, Gao Z, Asare AL, et al. Neutrophil-related gene expression and low-density granulocytes associated with disease activity and response to treatment in antineutrophil cytoplasmic antibody-associated vasculitis. Arthritis Rheumatol 2015;67:1922-32

34. Springer J, Villa-Forte A. Thrombosis in vasculitis. Curr Opin Rheumatol 2013;25:19-25.

35. Huang YM, Wang H, Wang C, Chen M, Zhao MH. Promotion of hypercoagulability in antineutrophil cytoplasmic antibody-associated vasculitis by c5a-induced tissue factor-expressing microparticles and neutrophil extracellular traps. Arthritis Rheumatol 2015;67:2780-90.
36. Willeke P, Kumpers P, Schluter B, Limani A, Becker H, Schotte H. Platelet counts as a biomarker in ANCA-associated vasculitis. Scand J Rheumatol 2015;44:302-8.

37. Miao D, Li DY, Chen M, Zhao MH. Platelets are activated in ANCA-associated vasculitis via thrombin-pars pathway and can activate the alternative complement pathway. Arthritis Res Ther 2017;19:252.

38. Zielinska KA, Van Moortel L, Opdenakker G, De Bosscher K, Van den Steen PE. Endothelial response to glucocorticoids in inflammatory diseases. Front Immunol 2016;7:592. 